\title{
Modeling the oxidation of low-density carbon fiber material based on micro-tomography
}

\author{
Joseph C. Ferguson ${ }^{\text {a }}$, Francesco Panerai ${ }^{\mathrm{a}, *}$, Jean Lachaud ${ }^{\mathrm{b}}$, Alexandre Martin ${ }^{\mathrm{a}}$, Sean C.C. Bailey ${ }^{\mathrm{a}}$, \\ Nagi N. Mansour ${ }^{\mathrm{c}}$ \\ ${ }^{a}$ Department of Mechanical Engineering, University of Kentucky, 151 Ralph G. Anderson Bldg., \\ Lexington, $K Y$ 40506, USA \\ ${ }^{b}$ Silicon Valley Initiatives, University of California Santa Cruz, NASA Ames Research Park Bldg. 19, \\ Moffett Field, CA 94035, USA \\ ${ }^{c}$ Advanced Supercomputing Division, NASA Ames Research Center, Mail Stop 258-1, Moffett Field, \\ CA 94035, USA
}

ABSTRACT: Oxidation is one of the main decomposition mechanisms of fibrous carbon/phenolic ablators employed in thermal protection systems for planetary entry capsules. The oxidation process is driven by two competing mechanisms: diffusion of reactants within the porous medium, and reaction rates at the surface of the fibers. These mechanisms are characterized by the Thiele number. Given that the Thiele number varies during an atmospheric entry, we aim to understand the effects of the diffusion/reaction processes on the decomposition of a porous carbon material in various regimes. We use a particle method for simulations of the oxidation process at microscale. The movement of oxygen reactants is simulated using a Brownian motion technique, and heterogeneous first-order reactions at the surface are modeled with a sticking probability law. To enable simulations of the fiber decomposition on actual materials, we use digitized computational grids obtained using X-ray microtomographic imaging. We present results for the oxidation of the substrate of the material used on the Mars Science Laboratory capsule that landed the Curiosity rover. We find that the depth of the reaction zone for this material is critically dependent on the Thiele number.

* Corresponding author. Tel.: +1 650604 3075. Email address: francesco.panerai@uky.edu (Francesco Panerai) 


\section{Introduction}

To reduce the cost of access to space and to address the challenges that will be faced by future exploration missions, NASA is optimizing thermal protection system (TPS) materials for atmospheric entry vehicles. Low-density carbon/phenolic ablators, built upon a rigid carbon fiber preform impregnated with phenolic resin, have proven to be a successful class of TPS materials for exploration missions. The flagship architecture within this class, is the Phenolic-Impregnated Carbon Ablator (PICA). This material was successfully used on the Mars Science Laboratory (MSL) and Stardust missions.

Carbon/phenolic ablators are designed to blow pyrolysis gases from the decomposing phenolic into the boundary layer formed at the surface of the material. The pyrolysis process mitigates part of the heat flux coming from the freestream plasma. Within the material, the fully charred (pyrolyzed) phenolic leaves a carbonized matrix. The carbonaceous remains, composed of carbon preform and charred phenolic, interact with the reactive species in the boundary layer through heterogeneous reactions. The main material recession processes in air are heterogeneous oxidation $\left(\mathrm{C}_{(\mathrm{s})}+\mathrm{O} \rightarrow \mathrm{CO}, \mathrm{C}_{(\mathrm{s})}+\mathrm{O}_{2} \rightarrow\right.$ $\mathrm{CO}_{2}$ ), phase changes (e.g. sublimation $\mathrm{C}_{(\mathrm{s})} \rightarrow \mathrm{C}$ ), and mechanical erosion by friction and shear stress (referred to as spallation). In this work we focus on heterogeneous oxidation processes. These are chief contributors to the exothermicity of the ablation zone, especially at the surface of the TPS, where the highly reactive matrix leaves the carbon preform exposed to incoming oxidants.

For highly porous ablators, where the reacting gases can percolate in-depth, it is important to look at the oxidation phenomenon at the scale of the fibers in order to understand the competing effects of diffusional mass transport and gas/surface reactions. A suitable approach for the analysis of this problem was proposed by Lachaud and Vignoles [1]. The same method was applied to study oxidation of carbon/phenolic ablators using artificially generated materials [2]. These studies used simplified digital models that describe the statistical three-dimensional (3D) morphology of composite material. Lachaud and Vignoles' method is based on a representation of the average gas diffusion by random walks, a sticking probability law for modeling heterogeneous reactions and a simplified marching cube algorithm for tracking the moving surface [1].

Using synthetic material models to mimic the microstructure of real composites has some limitations. Features of real materials, such as complex fiber morphologies, fiber clusters, actual pore-size distribution and other irregular characteristics, are very difficult to describe analytically and are not 
captured by ideal geometries. Synchrotron X-ray micro-tomography (micro-CT), used in the past to image $\mathrm{C} / \mathrm{C}$ composites [3], was recently applied to image substrates of carbon/phenolic ablators $[4,5]$. The technique provides a high-fidelity digital representation of the actual material microstructure where the geometry of the fibers is captured as a 3D matrix of grayscale values. Modern micro-CT instruments can reach voxel resolutions below a micrometer, thus are able to resolve the fibrous structures of carbon preform material in detail. The grayscale value within a voxel is proportional to the absorption of X-rays by the material, hence to the material density.

In this paper we aim to develop an understanding of the recession and in-depth evolution of the porosity of carbon fiber composites. We simulate the oxidation of FiberForm ${ }^{\mathrm{TM}}$ (Fiber Materials Inc.), a porous carbon fiber material made for commercial uses as furnace liners and for other applications. NASA has adopted FiberForm as the substrate for PICA. We refined the particle-based method described in [1], combined with high resolution micro-CT scans of the material, to study the oxidation of this substrate. The tomographic images of FiberForm were collected at the hard X-ray micro-CT beamline of the Advanced Light Source (ALS) at Lawrence Berkeley National Laboratory (LBNL). They consist of large datasets of the order of several billion voxels. In section 2, we describe the method of using micro-CT to develop a representation of the material on a three-dimensional Cartesian grid. These large datasets require dedicated tools for use in numerical simulations. To achieve this goal we have developed a computational framework, called PuMA for Porous Materials Analysis, described in section 3. The framework enables microscale simulations and calculations of certain material properties based on large micro-CT files.

In section 3.1, we describe the particle-based oxidation model. In section 3.2, we use analytical solutions for the oxidation of single fibers to verify the analysis tool. In section 4 , we study the oxidation of the fibrous material at various Thiele numbers (or regimes). In section 5, we conclude that the competing mechanisms of diffusion through the porous material and gas/surface reactions control the depth of the reaction volume, and propose future directions for research.

\section{Micro-tomography and visualization}

We used $14 \mathrm{keV} \mathrm{X-rays} \mathrm{generated} \mathrm{by} \mathrm{the} \mathrm{ALS} \mathrm{synchrotron} \mathrm{to} \mathrm{collect} \mathrm{micro-CT} \mathrm{projections} \mathrm{of}$ FiberForm. We adopted the ALS in-house procedure for tomographic reconstruction [6]. The digitized geometry of the material consists of grayscale voxels that are effectively a 3D X-ray image of the 
material that needs to be further interpreted. Figure 1 shows a surface rendering of the fibers in a 0.14 $\mathrm{mm}^{3}$ volume. The visualization was obtained by using OpenGL to render an approximation of the isosurface of the fiber geometry based on the marching cubes algorithm $[7,8]$.

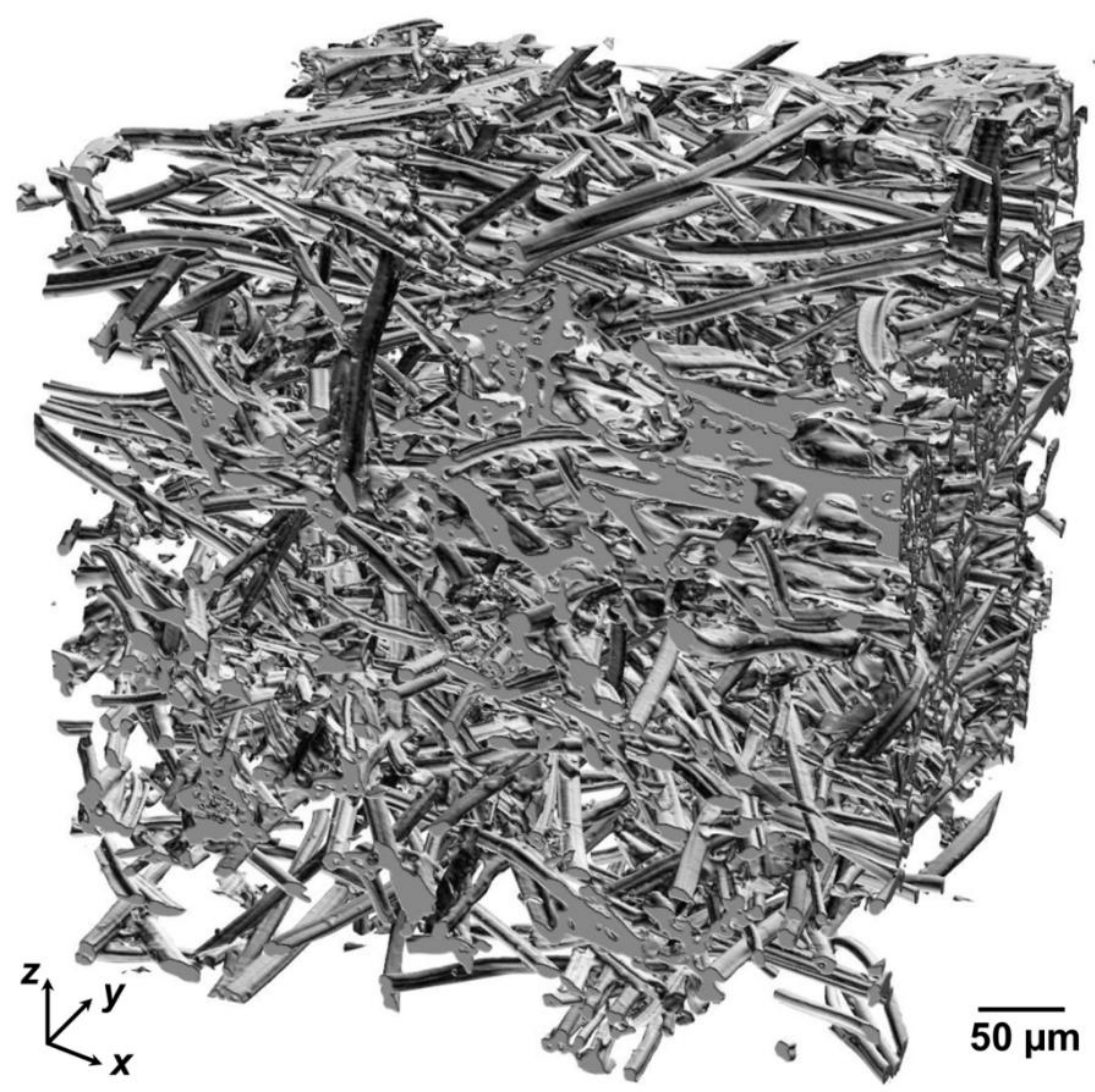

Fig. 1 - Volume rendering of FiberForm micro-CT. The image corresponds to a $0.14 \mathrm{~mm}^{3}$ cube with approximately $0.325 \mu \mathrm{m}$ voxel edge size (20× magnification). The rendering highlights the orthotropic structure of FiberForm, with fibers preferentially aligned within the $x-y$ plane. Fiber clusters and other irregular features are appropriately resolved.

The marching cubes algorithm is a level set method that effectively computes an isolevel surface of the grayscale levels. To select the cutoff level (or grayscale threshold, $\Gamma^{*}$ ) we start by computing a histogram of the grayscale distribution. Fig. 2 shows the histogram for the micro-CT image of FiberForm presented in Fig. 1. For this material, we find that the optimal grayscale cutoff is approximately the point of inflection on the histogram. This choice was confirmed by a quantitative comparison of the computed porosity from the image with the value specified by the material manufacturer, and a qualitative comparison of the tomography visualization with scanning electron 
microscopy images of virgin FiberForm. Any vertex with a grayscale value above this threshold is considered to be within the material, and any vertex below is considered void.

The marching cubes algorithm compares each voxel in the domain (represented by its 8 vertices) to a table of 256 possible polygon configurations, in order to determine which of the edge cases applies [7, 8]. The edge intersections of each triangle are then linearly interpolated from the vertex grayscale values. The resulting collection of triangles can be used for visualization as well as for computation of the model specific surface area.

To verify our implementation of the marching cubes algorithm in the PuMA framework, we generated grayscale images of analytical objects, and compared the computed surface area of their isosurface (calculated as a sum of the individual triangle areas) with the analytical surface area of these 3D shapes (e.g. cylinders and spheres).

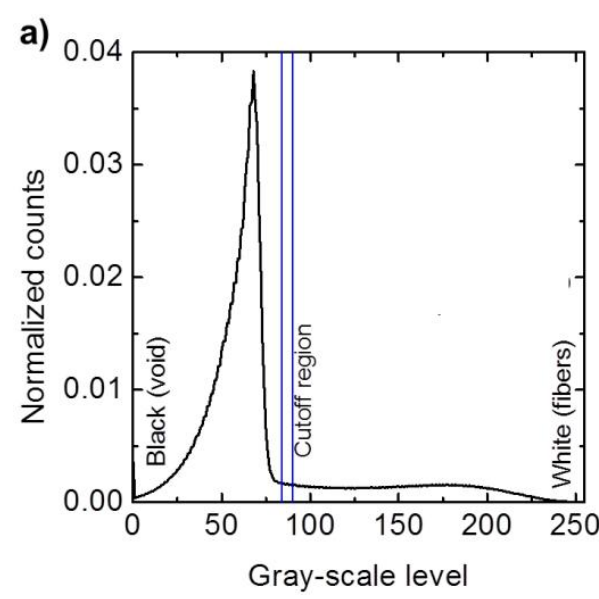

b)

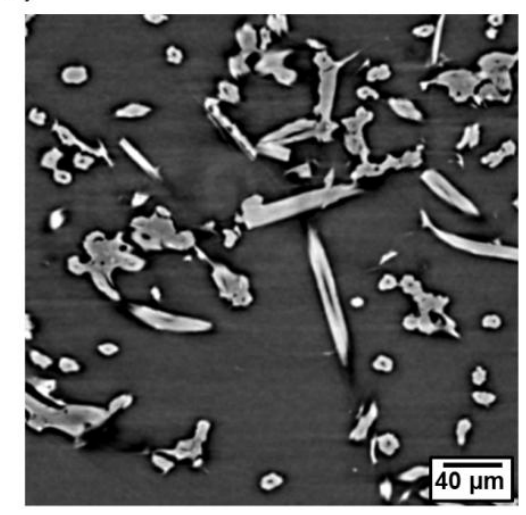

c)

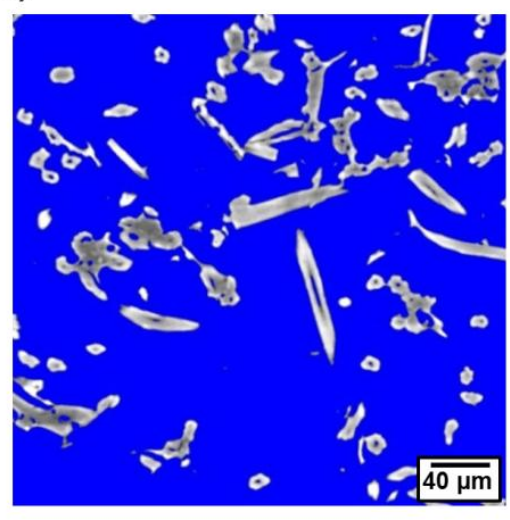

Fig. 2 - Segmentation of FiberForm micro-tomography dataset. a) Gray-scale histogram for dataset of Fig. 1. b) Example of a slice prior to segmentation. c) The same slice after segmentation, where the blue area represents the segmented void fraction.

\section{Microscale oxidation model and verification}

\subsection{Random walk oxidation}

\subsubsection{Digitization the micro-CT image of the material}

In the previous section we identified the fiber surface by defining a grayscale cutoff level. To digitize the geometry of the fibers, the $3 \mathrm{D}$ tomography array containing the grayscale values is processed as follows: 1) if a fiber surface passes through a voxel, the grayscale values of that voxel remain 
unchanged; 2) if the voxel is either completely outside or completely enclosed in the material, the voxel vertices are set to 0 or 255 , respectively. A schematic example of this processing is shown in Fig. 3, for a two-dimensional (2D) image. This modification is necessary in order to generate a digitized representation of the domain with a constant material density, since the material recession is based upon the reduction of voxel gray values. Normalizing to a uniform voxel density allows us to cope with the intrinsic variability of tomography, whereby equivalent volumes may result in datasets of different average grayscale, if different setting are used during X-ray scanning, during reconstruction, or during filtering and processing of the images. At the same time, it is also important that the digitized representation maintains surface integrity for collisions with oxygen. Since the vertex values of voxels containing an interface remain unchanged, the isosurface produced by the marching cubes algorithm remains unchanged between the two domains described by Fig. 3a and Fig. 3b.

During a simulation run, as the oxidation progresses, the grayscale values are modified to model the recession of the surface of the fibers, as described in section 3.1.4. The isolevel surface can be reapproximated and visualized at user-defined intervals, by reapplying the marching cubes algorithm.
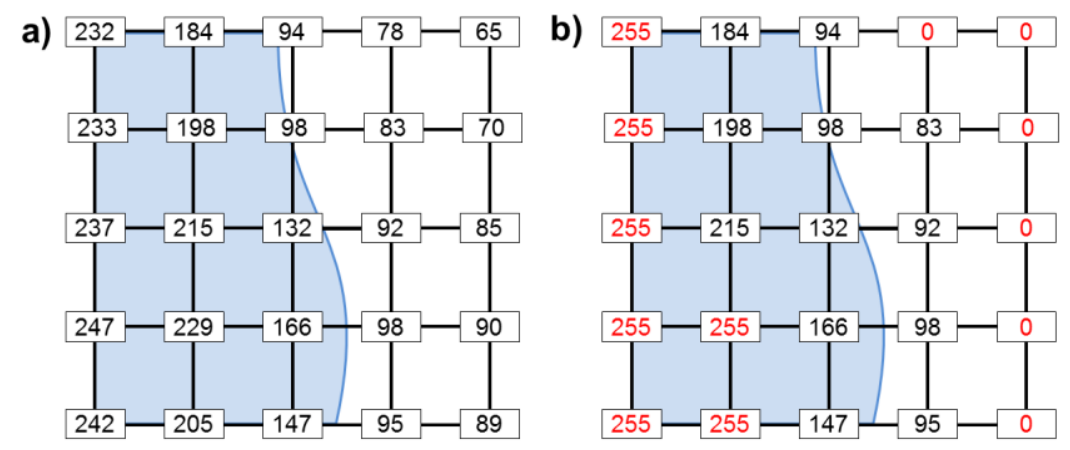

Fig. 3 - Example of the pre-processing of vertex grayscale values for a 2D image: a) original and b) processed image. The blue area represents carbon fiber, the white is the pore phase.

\subsubsection{Particle representation of the oxidizer}

The oxidizer reactants are represented by particles, called walkers. Each walker represents a userdefined number of oxidizer moles. In what follows we take oxygen as our oxidizer. A buffer zone of user-defined thickness is added at the top of the tomography domain. This buffer zone must be at least twice the characteristic length scale of the material [1]. A typical assumption for the characteristic length of FiberForm is the mean fiber diameter $\left(d_{f}\right)$ of approximately $11 \mu \mathrm{m}$. In the presented calculations a buffer zone of $30 \mu \mathrm{m}$ is added at the top of the domain. 
The buffer zone is set to maintain constant "atmospheric" conditions. Initially, it is populated with a number of walkers calculated as:

$$
N_{w}=\frac{y_{r} \rho_{g} V}{n_{w} M_{r}}
$$

where $y_{r}$ is the mass fraction of the reactant, $V$ is the volume of the buffer zone, $n_{w}$ is the user-defined number of moles per walker, $M_{r}$ is the molar mass of the reactant, and $\rho_{g}$ is the density of the gas mixture, calculated using the perfect gas law at temperature $T$ and pressure $p$ of the simulated conditions.

Once the number of walkers has been calculated, they are then randomly distributed throughout the buffer zone and begin their random walks. Whenever a walker leaves the buffer zone, a new walker is added at a random location within the buffer zone. Conversely, when a walker enters the buffer zone a randomly selected walker is removed. This ensures that the buffer zone maintains a fixed number of walkers, thus simulating constant atmospheric conditions. This technique satisfies a Dirichlet boundary condition on the upper part of the fluid phase. If a walker exits the top of the buffer zone (the side opposite the geometry), it is bounced back into the buffer zone. If a particle exits the computational domain (i.e. the tomography stack and the buffer zone) from the lateral walls, it is looped back to the opposite side of the domain, effectively emulating periodic boundary conditions at the lateral walls. In order to keep a constant diffusion length with respect to the top surface, the buffer zone is shifted downwards as the surface is oxidized. The shift is initiated by checking the number of oxidation events and the extent of oxidation in the top horizontal slice (the top plane of voxels in the tomography dataset). If this slice contains less than $0.001 \%$ material fraction, it is considered to be empty and the buffer zone is shifted down. At the same time interval, a filter is applied to isolated leftover material which might arise from numerical errors. If a vertex has a grayscale value above the threshold and all immediate neighbors are below the threshold, the material is removed. This prevents isolated fragments of material from impeding the shifting of the buffer zone.

\subsubsection{Modeling the diffusion of the oxidizer}

Oxygen diffusion is simulated using the random walk technique proposed by Lachaud and Vignoles [1]. The length of each walk $\Delta r$ affects the computational cost as well as the precision of the model [1]. A $\Delta r$ too small will result in increased computational costs. Conversely, a $\Delta r$ too large will result in a 
loss of accuracy. For the computations in this study, a parametric investigation was conducted to determine an appropriate value of $\Delta r$. It was found that a $\Delta r$ too large would result in incorrect fiber profiles for the analytical verification cases in section 3.2, as well as incorrect oxygen penetration depth for the results in section 4. For FiberForm it was found that a $\Delta r$ of $10 \mu \mathrm{m}$ (average fiber diameter) was sufficiently small to retain precision, and sufficiently large to allow time-effective simulations.

The direction of each walk in the 3D space is determined by choosing random polar angle $\theta$ and azimuth angle $\varphi$ in $[0,2 \pi]$, within the walker frame of reference. The unit direction vector is:

$$
\vec{u}=\langle\sin \theta \cos \varphi, \sin \theta \sin \varphi, \cos \theta\rangle
$$

The time increment of each walk, $\Delta t$ is calculated as follows:

$$
\Delta t=\frac{\Delta r^{2}}{6 D}
$$

where $D$ is the diffusion coefficient for the reactants mass transport.

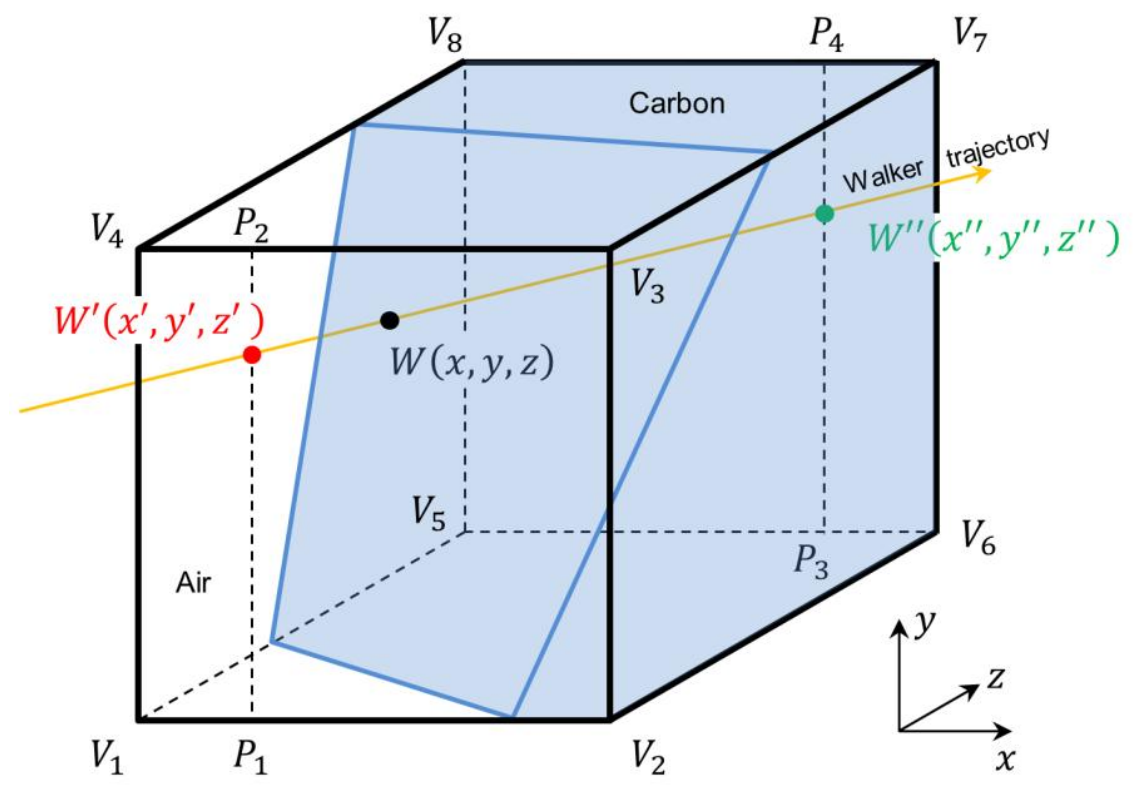

Fig. 4 - Schematic of the walker intersection with the carbon surface. The carbon phase is represented by the blue portion of the domain.

When walking the particle through the Cartesian grid, it must be determined whether a collision with the surface occurs at any point during the walk $\Delta r$. In previous implementations this was accomplished using simplified marching cubes [1,9], requiring frequent regeneration of the isosurface during an 
oxidation event. To improve performance and enable simulations on large micro-CT samples, an alternative method for surface collisions and recession was developed. For each voxel that the particle enters, the voxel is checked for the presence of a surface. If all eight vertices have values below the grayscale threshold level $\Gamma^{*}$, then the voxel is void of a surface and the particle continues its walk until the next voxel. If a surface is identified within the voxel, then the algorithm determines whether the path of the particle intersects with that surface. This is done by linearly interpolating the grayscale value at the entrance and exit points in the voxel. A schematic is presented in Fig. 4, where it is assumed that the walker enters the voxels at $W^{\prime}\left(x^{\prime}, y^{\prime}, z^{\prime}\right)$ in the surface defined by $V_{1}, \ldots, V_{4}$ and exit at $W^{\prime \prime}\left(x^{\prime \prime}, y^{\prime \prime}, z^{\prime \prime}\right)$ in the surface $V_{6}, \ldots, V_{8}$. If $\Gamma_{1}, \ldots, \Gamma_{8}$ are the gray values of the vertices $V_{1}, \ldots, V_{8}$, then the gray values $\Gamma^{\prime}$ at the entry location can be expressed as:

$$
\Gamma^{\prime}=a+b x^{\prime}+c y^{\prime}+d x^{\prime} y^{\prime}
$$

with $a=\Gamma_{1}, b=\Gamma_{2}-\Gamma_{1}, c=\Gamma_{4}-\Gamma_{1}, d=\Gamma_{1}-\Gamma_{2}+\Gamma_{3}-\Gamma_{4}$. An analogous expression can be written for $\Gamma^{\prime \prime}$ at the exit location. If $\Gamma^{\prime}<\Gamma^{*}$ and $\Gamma^{\prime \prime}>\Gamma^{*}$ a collision has occurred. The approximate location of this collision is determined through linear interpolation along the walker's path between the entrance and exit points:

$$
(x, y, z)=\left(x^{\prime}, y^{\prime}, z^{\prime}\right)+\frac{\Gamma^{*}-\Gamma^{\prime}}{\Gamma^{\prime \prime}-\Gamma^{\prime}}\left[\left(x^{\prime \prime}, y^{\prime \prime}, z^{\prime \prime}\right)-\left(x^{\prime}, y^{\prime}, z^{\prime}\right)\right]
$$

By using linear interpolation, the marching cubes algorithm is not needed during the oxidation simulation, and is only used for visualization purposes.

\subsubsection{Collision (sticking event)}

In the present algorithm, the collisions are modeled using a sticking event based method [1, 10]. The sticking probability is:

$$
P_{s}=\frac{1}{1+\frac{3 \gamma D}{2 k \Delta r_{w}}}
$$

where $D$ is the diffusion coefficient, $\Delta r_{w}$ is the distance from the start of the walk to the collision point at the surface, $\gamma$ is a correction coefficient to account for the non-Maxwellian nature of the random walk motion [1], and $k$ is the reactivity of the surface. 
To define a sticking event, a random number $\zeta \in[0,1]$ is generated from a uniform distribution. If $\zeta \geq P_{S}$, a sticking event does not occur and the particle is bounced back at a random angle for the remainder of its walk $\left(\delta r-\delta r_{w}\right)$. The random reflection vector reads the same as Eq. (2), with the conditions $\theta \in]-\pi / 2, \pi / 2[$ and $\varphi \in[0,2 \pi]$. Here $\theta$ is the angle between the random reflection and the normal to the surface and $\varphi$ is the azimuth of the random reflection in the plane tangential to the surface. The normal to the surface is approximated as a gradient of the voxel vertices.

If $\zeta<P_{S}$, then a sticking event occurs and the grayscale value $\Gamma$ of the lowest non-zero vertex in the voxel is reduced to $\Gamma-\alpha$. The grayscale attenuation $\alpha$ is computed as

$$
\alpha=\frac{M_{s} n_{w}}{\rho_{s} \tilde{V}} 256
$$

where $M_{S}$ and $\rho_{s}$ are the molar mass and the density of the solid (the carbon phase in case of FiberForm), and $\tilde{V}$ is the voxel volume. If $\alpha$ is higher than the grayscale value of the selected vertex, the next lowest non-zero vertex is reduced by the remaining attenuation value. Once a sticking event has occurred, the walker is removed.

\subsection{Verification}

An analytical solution presented in Lachaud et al. [1, 11] is used to verify the results of the oxidation model. In the analytical solution, a cylindrical fiber of specified radius $R_{f}$ and reactivity $k_{f}$ is surrounded by a matrix with a reactivity $k_{m}$ and is subjected to oxidation. It was shown that the profile of the resulting fiber at steady state can be calculated as a function of the reactivity contrast, $\mathrm{A}=$ $k_{m} \Omega_{m} / k_{f} \Omega_{f}$ and the Sherwood number $\mathrm{Sh}=k_{m} R_{f} / D$ [11]. Here the subscripts $m$ and $f$ indicate matrix and fiber respectively. $\Omega$ is the volume fraction and $D$ the diffusion coefficient.

Test cases were run at several combinations of A and Sh values in the reaction-limited and diffusionlimited regimes and compared to their analytical solutions. These cases were run on a domain with a cross-sectional size of $200 \times 200$ voxels and a fiber radius of 50 voxels, with voxel size of $1 \mu \mathrm{m}$. A fiber radius of 50 voxels was found to be large enough to converge to the exact solution with reasonable precision and a domain of $200 \times 200$ was large enough to maintain the correct oxygen concentration as a function of the diffusion length. $\Delta r$ was set to $10 \mu \mathrm{m}$ for the present calculations. Additional tests to verify the correctness of the solutions were performed with success using a $\Delta r$ up to $25 \mu \mathrm{m}$. 
The test cases are summarized in Fig. 5. Profiles of the interpolated cross sectional surface are compared to the exact solution to allow for a precise comparison. Figures 5a-d show that the model produces accurate results for a complete set of different regimes. Additional studies were performed to ensure that other parameters did not affect the steady state profiles. Maintaining a constant value for A and Sh, it was found that varying the walk distance, the radius of the fiber, and the number of oxygen moles per walkers affected the roughness of the steady state solution but not the overall fiber shape or height.

a) $A=10$, Sh $=0.1$

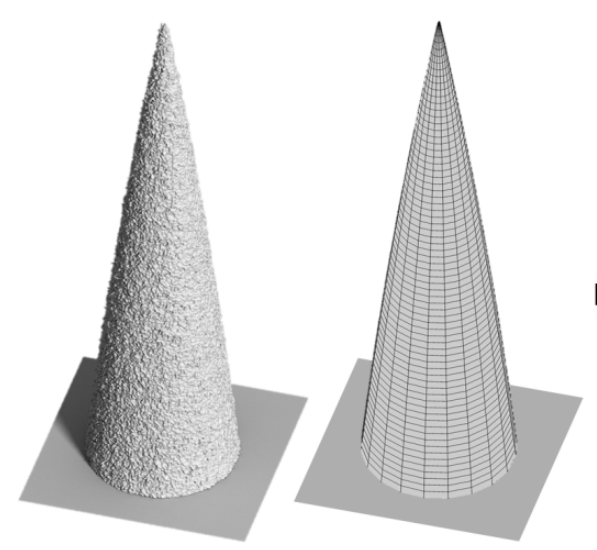

c) $\mathrm{A}=50, \mathrm{Sh}=0.5$

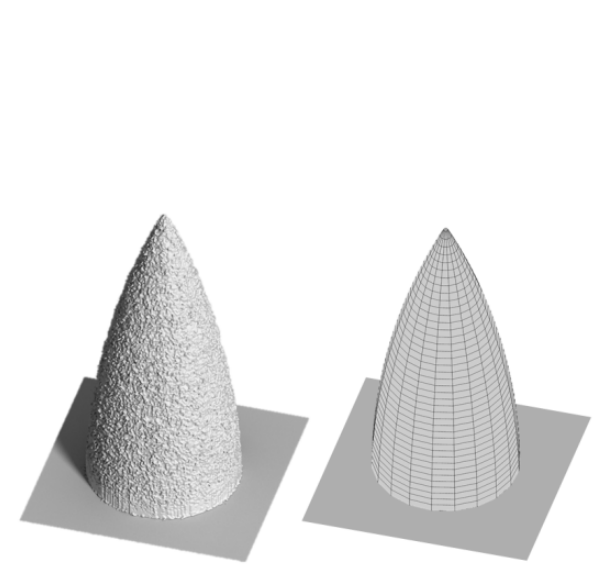

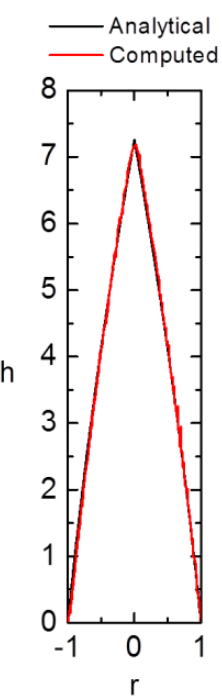

b) $A=2, S h=0.2$
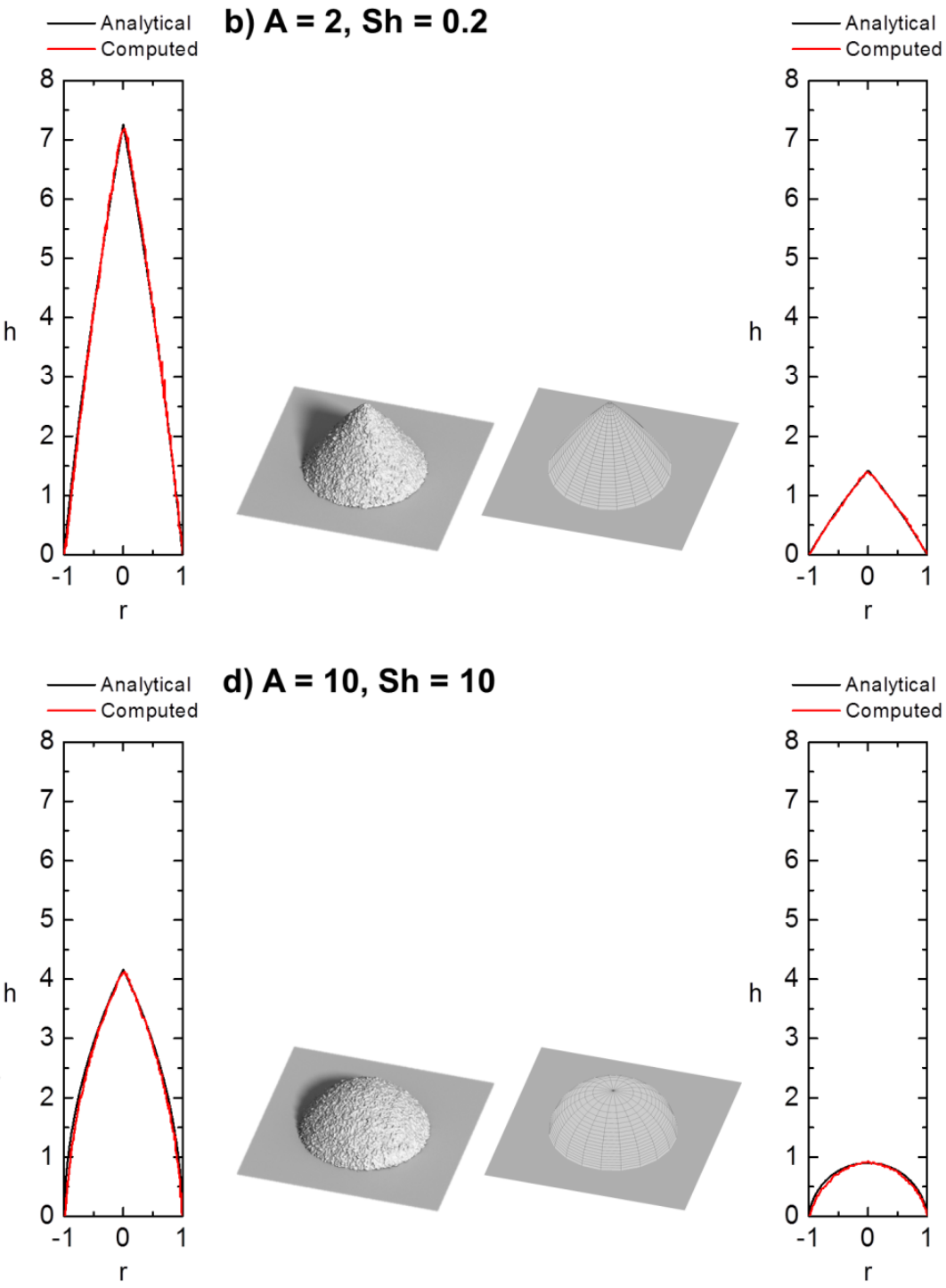

d) $A=10, S h=10$

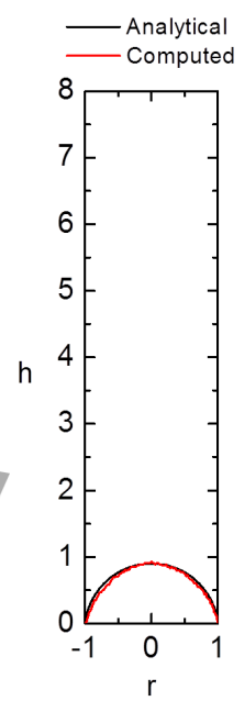

Fig. 5 - Test cases results for the verification of the microscale oxidation model implemented in PuMA. Analytical solutions for different reactivity contrast A and Sherwood number Sh are computed according to [11]. 


\section{Results and discussion}

Using the model described in the previous section, we simulated the oxidation of FiberForm. In order to study the competition between diffusion and reaction processes within the porous material, we performed oxidation simulations at different Thiele regimes. The Thiele number is defined as:

$$
\Phi=\frac{L}{\sqrt{\mathcal{D}_{\text {eff }} /\left(s_{f} k_{f}\right)}}
$$

where $L$ is a characteristic length scale of the system, $\mathcal{D}_{\text {eff }}$ is the effective diffusion coefficient, $s_{f}$ is the specific surface of the porous medium and $k_{f}$ is the fiber reactivity. $\Phi$ describes the competition between reactant diffusion and surface reactivity in a porous medium at a characteristic scale $L$. In the case of oxidation, high Thiele numbers characterize a diffusion-limited regime, where the decomposition of the fibers occurs mostly at the surface of the material. Conversely, at low Thiele numbers, the oxidation is a volumetric, reaction-limited process. Oxygen can penetrate in depth and a decomposition zone larger than the fiber scale can be observed. At intermediate Thiele numbers, the scale of diffusion and reaction are similar. In such mixed regime, the reaction zone is of the order of the fiber scale.

In Fig. 6 we present the results of oxidation simulations performed with our method using the sample displayed in Fig. 1 as the computational domain. In order to reduce the computational cost, we downsampled the domain by a factor of 2 , giving a voxel size of approximately $0.65 \mu \mathrm{m}$. The resulting dataset, with slightly over half billion voxels, maintains most of the information of the unscaled domain and can be handled with a memory of less than 1 gigabyte. The time scale $\tau$ in Fig. 6 (and also in the following Fig. 7) is a nondimensional unit describing the oxidation time. Each frame is taken at increments of approximately $20 \%$ of the total oxidation time for the given regime.

For runs in the volumetric ablation regime, the sticking probability for each collision event was very low, of the order of $10^{-7}$. This resulted in very large run times to oxidize the complete volume. To avoid this, the steady state oxygen concentration, as a function of distance from the buffer zone, was determined, using the normal procedure described in section 3. The volumetric ablation cases were then performed with a high sticking probability, but distributing the oxygen walkers throughout the voids of the material at the computed oxygen concentration, rather than placing them in the buffer zone and letting them diffuse. This is not an ideal solution, but it provides an adequate solution until the model 
can be parallelized to speed up the computational performance of $P u M A$ and allow use of supercomputing facilities.

a)

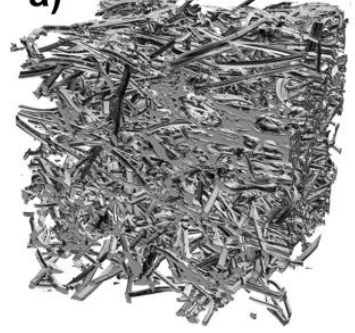

b)

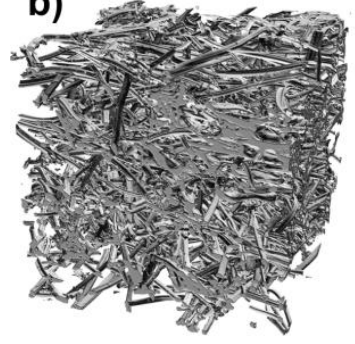

c)

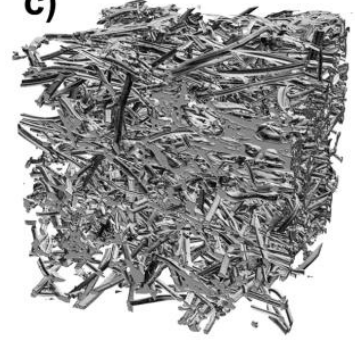

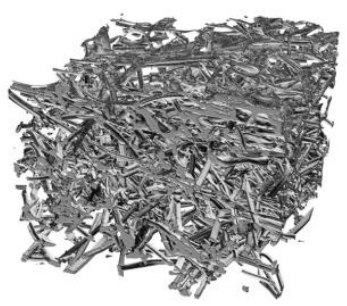
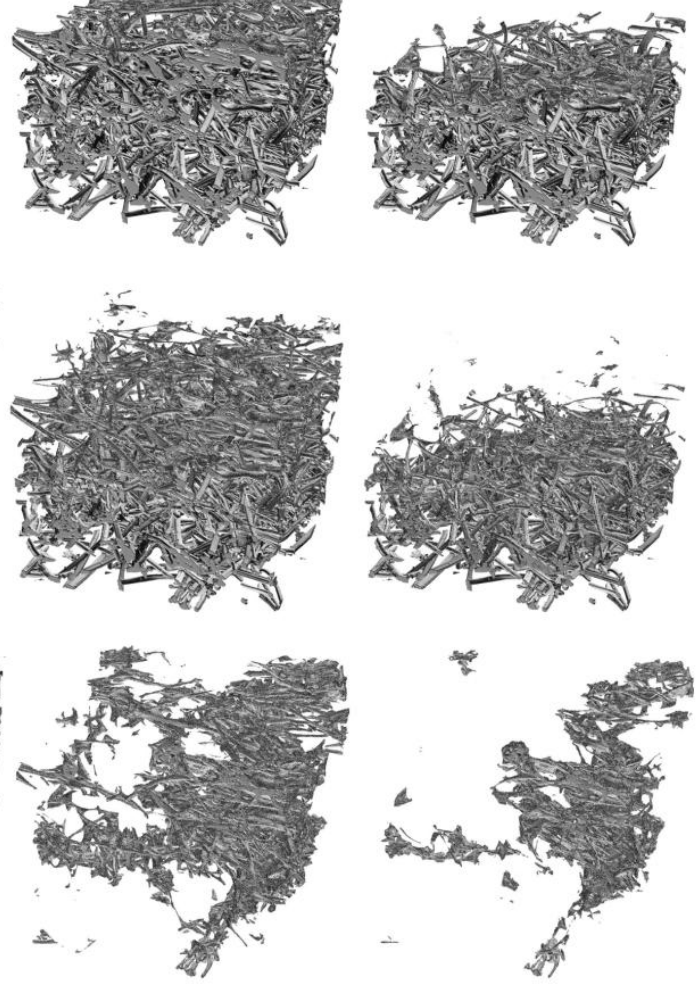
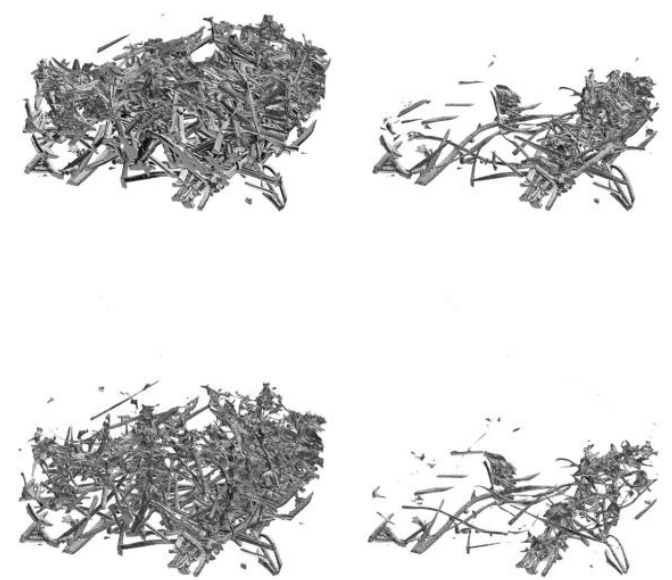

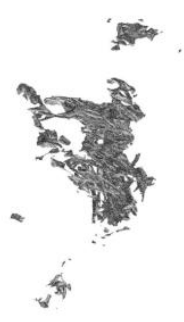

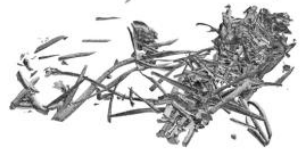

$\boldsymbol{\tau}$

Fig. 6 - Rendering of the progressive oxidation of FiberForm under different regimes: a) surface oxidation regime $(\Phi \gtrsim 50), b)$ mixed regime $(\Phi \approx 1)$ and c) volume oxidation regime $(\Phi \lesssim 0.05)$.

The renderings in Fig. 6 show that the model is able to capture the physics of different conditions, whereby only surface fibers are oxidized (Fig. 6a) in the diffusion-limited regime, while the material is oxidized in depth (Fig. 6c) in the reaction-limited case.

Figure 6b, presents the intermediate case where oxidation competes directly with the diffusion process at a Thiele number close to 1 . Oxygen diffuses in the preform material but, at the same time, it is quickly consumed by the oxidation reaction of the carbon fibers.

The surface oxidation regime is typically experienced during arcjet testing at high heat fluxes. When a lightweight carbon/phenolic ablator like PICA is exposed to a high enthalpy flow in an arcjet 
environment, the phenolic phase, which is more reactive than the carbon fibers, decomposes via pyrolysis processes and leaves the carbon fibers of the substrate exposed to the freestream reactants. At very high temperatures (> $2000 \mathrm{~K}$ ) the reactivity of the carbon fibers to oxygen is very high. Therefore the rate of consumption of the reactants is much higher than the rate of diffusion through the porous medium. In this situation, most of the incoming oxygen is consumed near the surface and the process leads to materials recession. Under those conditions, the carbon fibers at the surface tend to decompose into a characteristic needle shape, as it was observed during analysis of carbon/phenolic samples tested at high enthalpy $[12,13]$. Similar to arcjet conditions, surface oxidation occurs at the peak heating point along the trajectory of an atmospheric entry capsule.

Volume oxidation and mixed regime conditions were observed during flow-tube reactor experiments performed at NASA Ames Research Center [14, 15]. During those experiments, FiberForm samples were heated to temperatures between 700 and $1300 \mathrm{~K}$, while being subjected to an oxidizing airflow at controlled rate. Low pressures $(\leq 10 \mathrm{kPa})$ and low temperatures $(\leq 900 \mathrm{~K})$ promoted a reaction-limited regime in the porous medium, in which case oxidation is mostly a volumetric process. In-depth decomposition was observed in scanning electron micrographs of the material after high temperature exposure. Thiele numbers close to 1 (the mixed regime) were estimated for temperatures between 1000 and $1300 \mathrm{~K}$ and pressures above $10 \mathrm{kPa}$. Volume ablation and mixed regimes are typical of the late phases of an entry trajectory, when the material has cooled down to relatively low temperatures (below $\approx 1000 \mathrm{~K}$ ). Under such conditions the recession is minimal, however the material structure can be significantly weakened in depth, augmenting material loss due to spallation, shear and vibration effects. 


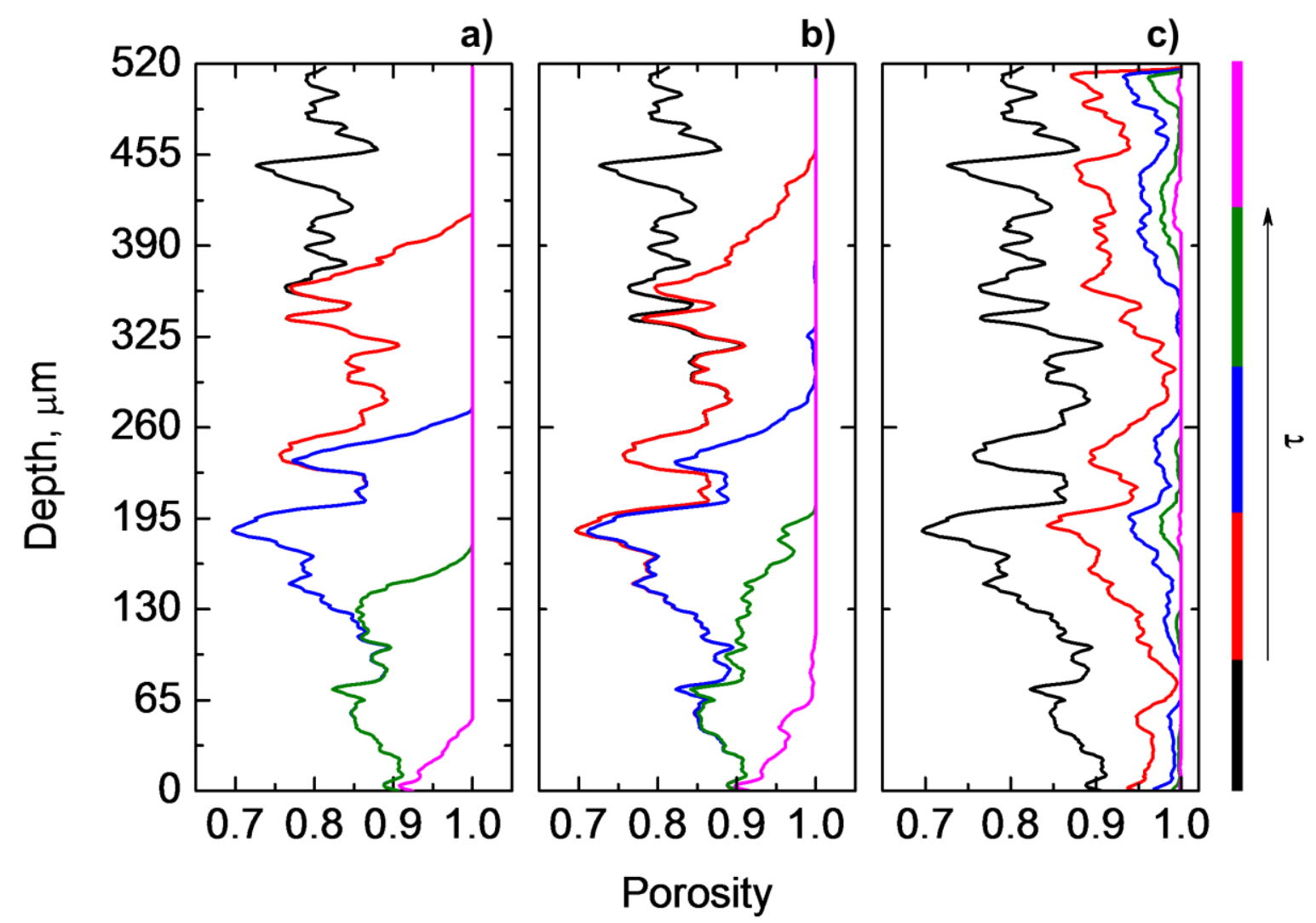

Fig. 7 - Evolution of the in-depth a) surface oxidation regime $(\Phi \gtrsim 50), b)$ mixed regime $(\Phi \approx 1)$ and c) volume oxidation regime $(\Phi \lesssim 0.05)$.

In order to assess the depth of the reaction zone for the PuMA simulations, we present in Fig. 7 indepth (z-direction) distribution of porosity at five time instants for three Thiele numbers. The porosity is computed as the void fraction of each slice. The oxidation depth can be estimated by calculating the distance between the location where the porosity of the sample is 1 (fully oxidized) and the location where the porosity profile deviates from that of the virgin material. Alternatively, it can be determined based on the in-depth oxygen distribution. From the figure, we estimate an oxidation depth below 65 $\mu \mathrm{m}$ in surface oxidation regime, while the reaction zone extends to the full depth (520 $\mu \mathrm{m}$ in this case) in the volume oxidation regime. It should be noted that for an inhomogeneous material such as FiberForm, the bulk material porosity may significantly vary for microscale samples. Therefore, the oxidation depth, which strongly depends on the local porosity of the material, will also vary based on the region selected. 
An additional study was conducted to examine the effect of the Thiele regime on the oxidation depth. We selected a $500 \mu \mathrm{m}$ thick microscale sample and six Thiele regimes between $\Phi=0.01$ and $\Phi=10$ encompassing the surface, mixed, and volume oxidation regimes. The oxidation simulation was run until the oxidation depth reached steady state, then the in-depth oxygen distribution $C_{z}$ was recorded. The results are shown in Fig. 8, where $C_{z}$ is normalized by the concentration in the buffer zone $C^{*}$. It can be observed that Thiele number is a strong indicator of the oxygen penetration. The oxidation depth will depend on the ability of the oxygen to penetrate through the porous medium and, in a real case, also on the in-depth temperature gradient. In reaction-limited oxidation, for any $\Phi \lesssim 0.5$, significant amount oxygen is present throughout the whole domain. Hence, the in-depth extent of oxidation will be driven by the local temperature of the material.

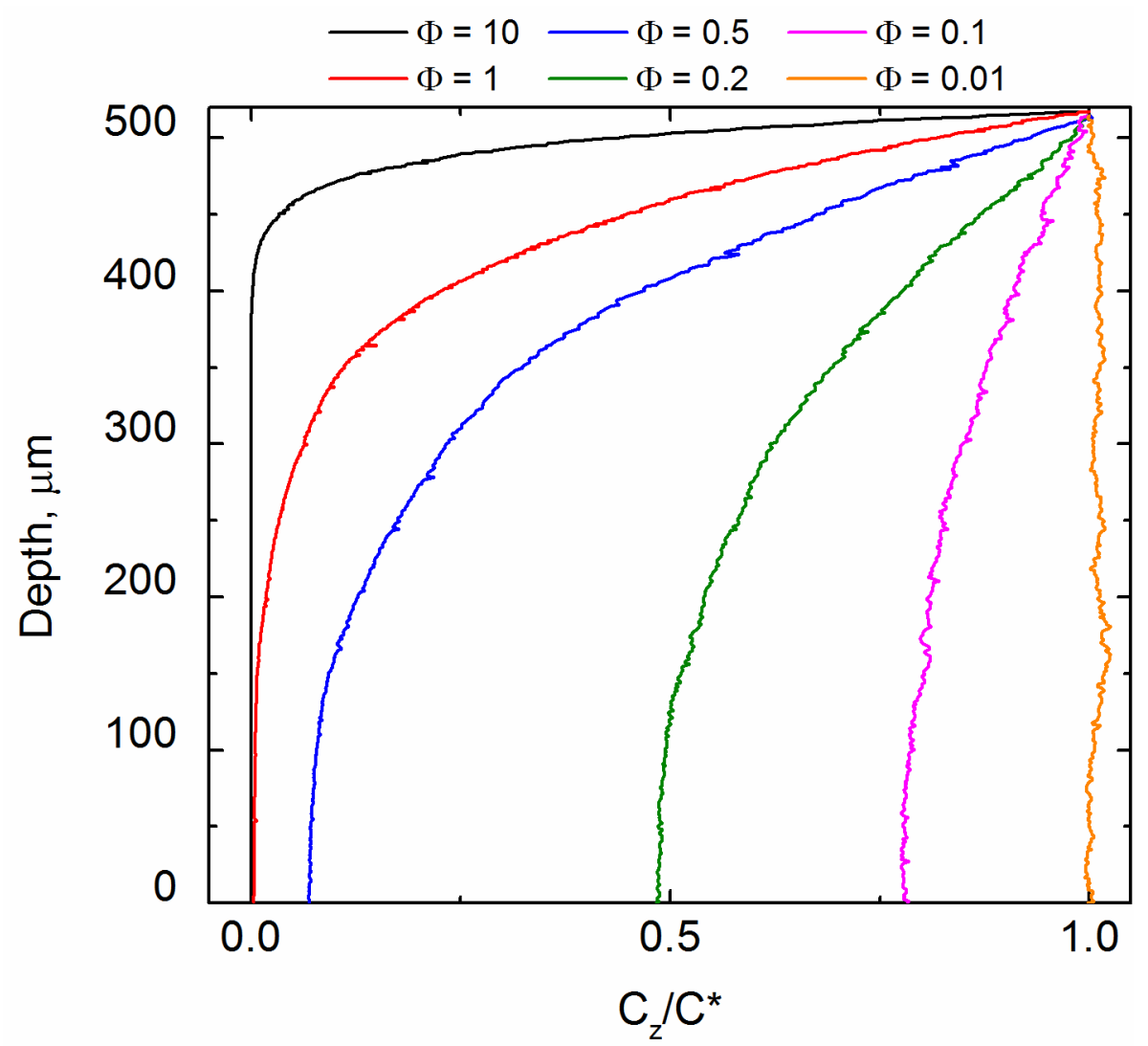

Fig. 8 - In-depth oxygen concentration for different Thiele regimes.

\section{Comparison with experiments}

The results shown in Section 3 verified the implementation of the oxidation model in $P u M A$ and the simulations presented in Section 4 showed the ability of the model to predict different oxidation 
regimes. Beyond these numerical experiments, simulations were compared to results from highenthalpy experiments of FiberForm. Micro-CT images were collected from a sample of FiberForm that had been subjected to arcjet high-enthalpy airflow, imposing $200 \mathrm{~W} / \mathrm{cm}^{2}$ heat flux and $0.03 \mathrm{~atm}$ at the surface of the material. Under these conditions, the material experienced a surface temperature of 2395 K. Using the same parameters described in Section 2, a micro-CT scan of the plasma-decomposed sample was obtained for a region extending from the front surface to approximately $400 \mu \mathrm{m}$ in-depth, in the vicinity of the stagnation point.

a)

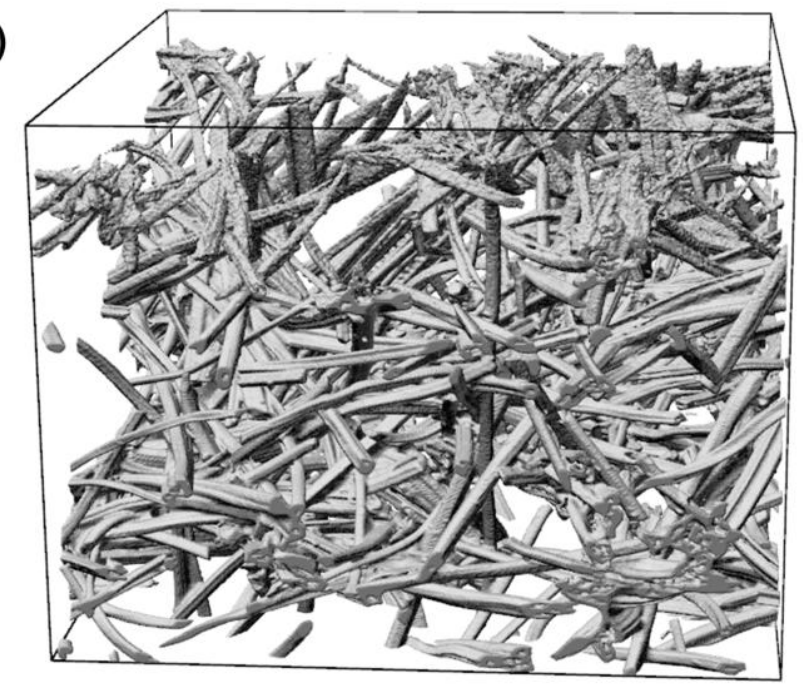

b)

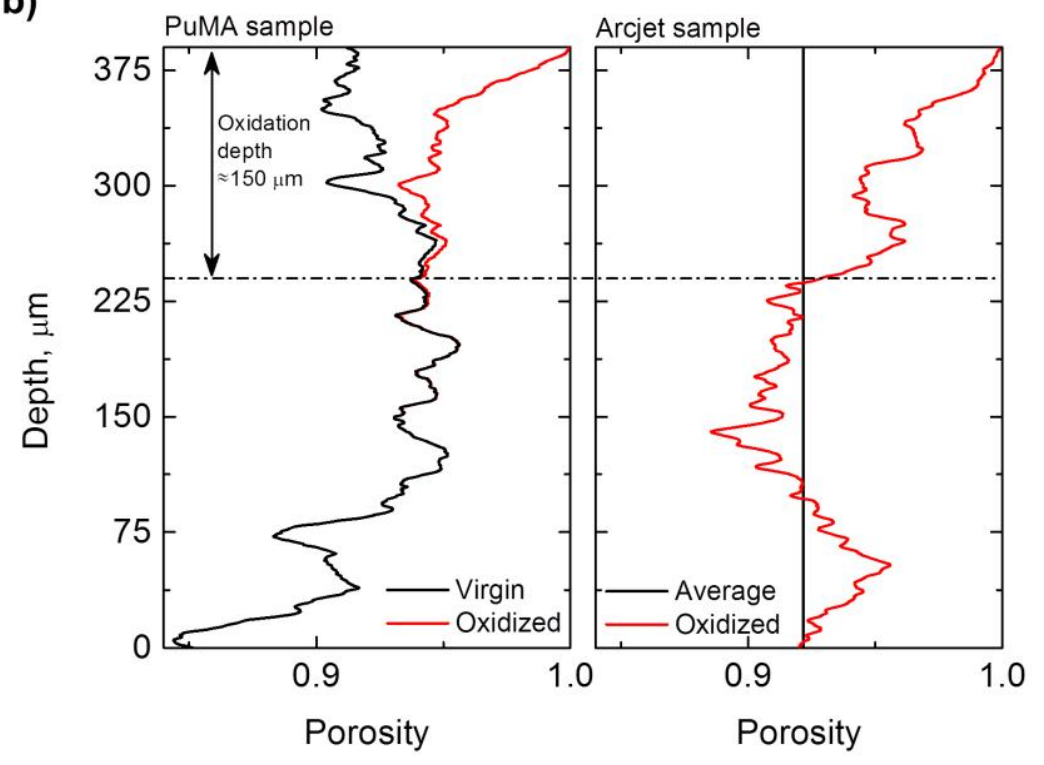

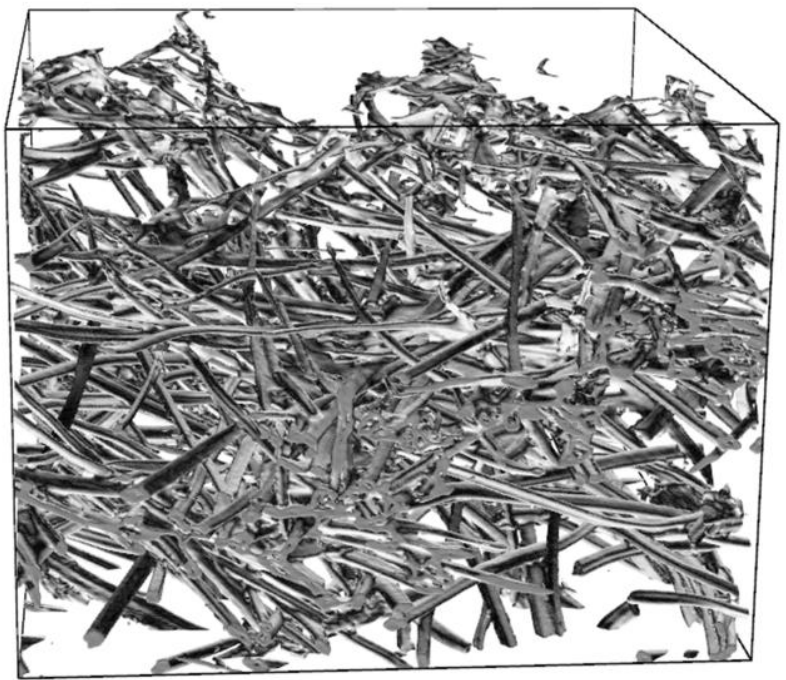

c)

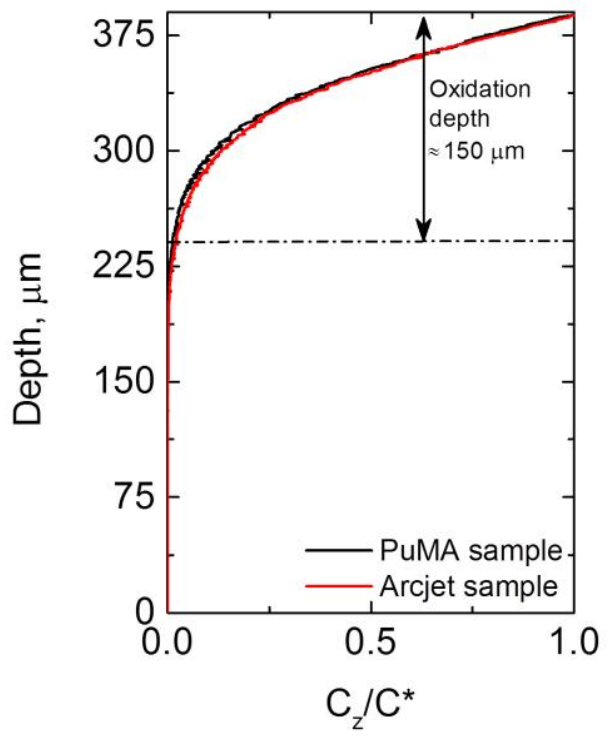

Fig. 9 - Comparative analysis of simulation with experiment on FiberForm sample exposed to arcjet airflow. a) Visualization of PuMA oxidation (left) and micro-CT of arcjet sample (right). b) Porosity as a function of material 
depth for the two cases, allowing the identification of the oxidation depth. c) Normalized, in-depth reactant concentration through the porous media.

An oxidation simulation was performed under the same conditions of the arcjet test, starting from a micro-CT volume of the virgin FiberForm. Once steady-state conditions for the oxidation depth were achieved in the PuMA simulation, a comparison of the two was performed. The results are summarized in Fig. 9. Observing the micro-CT rendering of the two models, it can been seen (Fig. 9a) that the oxidized fibers have similar shape and characteristics between the two data sets. Of interest is the observation of the typical needle shape that occurs during diffusion-limited oxidation. The oxidation depth for both samples is shown in Fig. 9b, by plotting the porosity as a function of material depth. It is noted that there is a large variability in the porosity at this scale. For the PuMA-oxidized sample, the oxidation depth was determined by the location where the in-depth porosity profile of the oxidized volume deviates from that of the virgin one. This procedure could not be used for the micro-CT of the arcjet case given that the image of the virgin sample is not available. The oxidation depth was determined in this case by the location where the in-depth porosity profile deviates from the average porosity value. The oxidation depth was determined to be $\approx 150 \mu \mathrm{m}$, with close agreement between simulations and experiment.

A further verification was performed by comparing the normalized in-depth reactant concentration $C_{z} / C^{*}$ for the two cases (with $C^{*}$ being the buffer zone concentration) in Fig. 9c. To determine $C$ for the arcjet model, a random-walk simulation was performed, allowing for the walker to react with the surface, while impeding surface recession. The oxygen concentration as a function of material depth was recorded once it reached steady state. As shown in Fig. 9c the penetration through the numericallyoxidized material closely replicates that of the arcjet model and provides additional support for the estimated oxidation depth of $\approx 150 \mu \mathrm{m}$. This value is also consistent with the analysis of a similar carbon preform subjected to ablation in induction-plasma flows [12].

The present model is suitable to replicate the overall diffusion/reaction mechanisms and predict the average oxidation of the fibers, as shown by the good agreement between experiments and simulations. The minimum voxels size achievable by our micro-CT images $(\sim 0.3 \mu \mathrm{m})$ is not sufficient to fully resolve features of the order of a micron or less. It is well known that depending on the oxidation conditions and the type of carbon fiber, different oxidation mechanisms can occur, leading to different, localized oxidation rates and sometimes showing small scale features. For example, in flow-tube 
oxidation experiments, FiberForm shows pitting of the fibers that increases the surface roughness and modifies the local reactive surface area of the material [14]. The material removal predicted by our micro-CT-based simulations increases the roughness of the fibers, but does not capture the pitting phenomenon. This is due to the fact that surface chemistry at the atomic scale and heat transfer processes within the material are not included. The model considers a larger scale and therefore encompasses any locally averaged oxidation rate.

In addition, for carbon fibers interacting with high-speed flows, possible contributions to material decomposition come from mechanical removal due to shear and friction. Spallation effects, not included in the present model, enhance material removal compared to a static oxidizing atmosphere.

The addition of heat transfer, surface chemistry and mechanical decomposition models to micro-CTbased simulations will be considered in future studies.

\section{Conclusions}

We presented a model for simulating the microscale oxidation of low-density carbon-fiber composites, based on high fidelity imaging of the material microstructure. This model, implemented in the PuMA framework, is able to import synchrotron micro-CT data and use them as computational grids for simulations and material properties calculations. We showed that the competing mechanisms of diffusion though the porous material and gas/surface reactions control the depth of the reaction volume. We can speculate from Fig. 6 that the amount of spallation (loose fibers) will be a strong function of Thiele number. The suitability of tomography domains to accurately quantify material decomposition by mechanical effects as shear and friction will be the subject of future investigations.

Comparing simulations with micro-CT data of oxidized FiberForm from plasma testing, we showed the ability of the model to predict in-depth oxidation. For a diffusion-limited case we found an oxidation depth of $\approx 150 \mu \mathrm{m}$, consistent with previous investigations. The method can be used to determine hightemperature effective reactivities for carbon under arcjet conditions.

We point out that the availability of an accurate, 3D description of the material microstructure will enable computations of the orthotropic properties such as thermal conductivity, permeability and tortuosity, for FiberForm and other ablative thermal protection materials. Calculations of such properties at high temperatures, based on tomography measurements, have been proposed by past investigations on porous ceramics [16-18]. Recent progress for carbon ablators has been shown in [19]. 
In addition, the methodology presented here could be easily ported to more complex particle-based methods, such as Direct Simulation Monte Carlo. Having detailed representation of the oxidizing gas will enable the introduction of complex reaction models for the gas/surface interactions at the fiber surface. Particularly important is tracking the generation of $\mathrm{CO}$ and $\mathrm{CO}_{2}$ oxidation products and their possible reactions with carbon fibers under high temperature.

\section{Acknowledgements}

The authors are indebted to T.A. Sandstrom for his assistance in the development of the PuMA framework. A.A. McDowell and D.Y. Parkinson are gratefully acknowledged for their help in the LBNL/NASA Ames collaboration on micro-CT measurements. This work was performed under the Entry System Modeling Project (M.J. Wright program manager) of the NASA Game Changing Development (GCD) Program. The work was partly supported by NASA SBIR Phase II Award NNX10CC53P, NASA and Kentucky EPSCoR Award NNX13AN04A, NASA Award NNX14AI97G, and NASA Kentucky under NASA Award NNX10AL96H. The Advanced Light Source is supported by the Director, Office of Science, Office of Basic Energy Sciences, of the U.S. Department of Energy under Contract No. DE-AC02-05CH11231.

\section{References}

[1] Lachaud J, Vignoles GL. A Brownian motion technique to simulate gasification and its application to C/C composite ablation. Computational Materials Science. 2009;44(4):1034-41.

[2] Lachaud J, Cozmuta I, Mansour NN. Multiscale Approach to Ablation Modeling of Phenolic Impregnated Carbon Ablators. J Spacecraft Rock. 2010;47(6):910-21.

[3] Vignoles GL. Image segmentation for phase-contrast hard X-ray CMT of C/C composites. Carbon. 2001;39(2):167-73.

[4] Mansour NN, Panerai F, Martin A, Parkinson DY, MacDowell AA, Fast A, et al. A new approach to light-weight ablators analysis: from micro-tomography measurements to statistical analysis and modeling. $44^{\text {th }}$ AIAA Thermophysics Conference, 2013, AIAA 2013-2768.

[5] Panerai F. Microscale Modeling and Characterization of Carbon-Based Ablators Decomposition. Radiation and gas-surface interaction phenomena in high-speed reentry. Urbana-Champaign, IL, USA, 
2014. NATO-STO-VKI Lecture Series, STO AVT-218: von Karman Institute for Fluid Dynamics; Paper No. 8.

[6] MacDowell AA, Parkinson DY, Haboub A, Schaible E, Nasiatka JR, Yee CA, et al. X-ray microtomography at the Advanced Light Source. SPIE Proceedings Vol. 8506: Developments in X-Ray Tomography VIII, 2012.

[7] Lorensen WE, Cline HE. Marching cubes: A high resolution 3D surface construction algorithm. SIGGRAPH Comput Graph. 1987;21(4):163-9.

[8] Watt AH, Watt M. Advanced Animation and Rendering Techniques: Theory and Practice: ACM Press; 1992.

[9] Vignoles GL, Donias M, Mulat C, Germain C, Delesse J-F. Simplified marching cubes: An efficient discretization scheme for simulations of deposition/ablation in complex media. Computational Materials Science. 2011;50(3):893-902.

[10] Sallès J, Thovert JF, Adler PM. Deposition in porous media and clogging. Chem Eng Sci. 1993;48(16):2839-58.

[11] Lachaud J, Aspa Y, Vignoles GL. Analytical modeling of the steady state ablation of a 3D C/C composite. Int J Heat Mass Transfer. 2008;51(9-10):2614-27.

[12] Helber B, Chazot O, Hubin A, Magin TE. Microstructure and gas-surface interaction studies of a low-density carbon-bonded carbon fiber composite in atmospheric entry plasmas. Composites Part A: Applied Science and Manufacturing. 2015;72(0):96-107.

[13] Vignoles GL, Lachaud J, Aspa Y, Goyhénèche J-M. Ablation of carbon-based materials: Multiscale roughness modelling. Compos Sci Technol. 2009;69(9):1470-7.

[14] Panerai F, Martin A, Mansour NN, Sepka SA, Lachaud J. Flow-Tube Oxidation Experiments on the Carbon Preform of a Phenolic-Impregnated Carbon Ablator. J Thermophys Heat Transfer. 2014;28(2):181-90.

[15] Panerai F, Martin A, Lachaud J, Mansour NN. Experimental and numerical study of carbon fiber oxidation. 52 ${ }^{\text {nd }}$ Aerospace Sciences Meeting 2014, AIAA 2014-1208. 
[16] Petrasch J, Meier F, Friess H, Steinfeld A. Tomography based determination of permeability, Dupuit-Forchheimer coefficient, and interfacial heat transfer coefficient in reticulate porous ceramics. International Journal of Heat and Fluid Flow. 2008;29(1):315-26.

[17] Haussener S, Coray P, Lipiński W, Wyss P, Steinfeld A. Tomography-Based Heat and Mass Transfer Characterization of Reticulate Porous Ceramics for High-Temperature Processing. J Heat Transfer. 2009;132(2):023305.

[18] Haussener S, Jerjen I, Wyss P, Steinfeld A. Tomography-Based Determination of Effective Transport Properties for Reacting Porous Media. J Heat Transfer. 2011;134(1):012601.

[19] Panerai F, Ferguson J, Lachaud J, Martin A, Gasch MJ, Mansour NN. Analysis of fibrous felts for flexible ablators using synchrotron hard X-ray micro-tomography. $8^{\text {th }}$ European Symposium on Aerothermodynamics for Space Vehicles, 2015, Paper N. 89747. 